\title{
Respostas neuromusculares e metabólicas do método de treinamento de força FST-7 em homens treinados
}

http://dx.doi.org/10.11606/1807-5509202000030437

\section{Resumo}

0 objetivo deste estudo foi avaliar as respostas agudas neuromusculares e metabólicas do método de treinamento de força (TF) FST-7. Para tal, 10 homens experientes em TF foram submetidos a três protocolos experimentais. 1) FST-7 (sete séries de 10 repetições de extensão isocinética de joelho com 30 s de intervalo entre as séries, incluindo 20 - $25 \mathrm{~s}$ de alongamento do quadríceps); 2) Controle (CON, sete séries de 10 repetições de extensão isocinética de joelho com $30 \mathrm{~s}$ de intervalo entre as séries, sem alongamento entre as séries); e 3) Tradicional (TRAD, sete séries de 10 repetições de extensão isocinética de joelho com $120 \mathrm{~s}$ de intervalo entre as séries, sem alongamento entre as séries). 0 trabalho total do TRAD $(12.160 \pm 2.200$ J) foi maior $(p<0,05)$ que o FST-7 $(9.361 \pm 1.714 \mathrm{~J})$ e CON $(10.730 \pm 2.360 \mathrm{~J})$, CON também produziu trabalho total maior que FST-7 $(p<0,05)$. A queda no trabalho entre as séries (índice de fadiga) foi maior $(p<0,05)$ no FST-7 $(38,5 \pm 13,4 \%)$ em comparação ao CON $(29,0 \pm 10,9 \%)$ e TRAD $(12,0 \pm 10,4 \%)$. A espessura muscular e o lactato sanguíneo aumentaram significativamente $(p<0,05)$, no entanto sem diferença entre os três protocolos $(p>0,05)$. Estes resultados sugerem que o FST-7 apresenta respostas metabólicas similares aos métodos CON e TRAD, no entanto o estresse mecânico imposto pelos métodos TRAD e CON foram superiores ao FST-7.

PalAVRAS-CHAVE: Exercício resistido; Alongamento; Hiperemia muscular; Índice de fadiga; Lactato sanguíneo.
*Faculdade de Educação Física

Universidade de Brasília, Brasília, DF, Brasil.

**Universidade Federal de Ouro Preto, Ouro Preto, MG, Brasil.

${ }^{* * *}$ Centro Universitário, do Distrito Federal,

Brasília, DF, Brasil

\section{Introdução}

Níveis adequados de força e massa muscular são fundamentais para saúde e qualidade de vida da populaçáo em geral, bem como podem ser determinantes para o desempenho de atletas ${ }^{1,2}$. Nesse sentido, o treinamento de força (TF) tem sido proposto como uma das formas mais eficientes de maximizar os ganhos de força e hipertrofia muscular ${ }^{3}$, ${ }^{4}$. A partir desta lógica, diferentes métodos de TF têm sido desenvolvidos com o objetivo de otimizar estes ganhos neuromusculares ${ }^{5-7}$. Esses métodos, basicamente, consistem na manipulação das variáveis do TF (p. ex., intensidade, volume, intervalo de recuperação) para o aumento progressivo do estresse mecânico ou metabólico. Todavia, é importante destacar que a eficácia de muitos desses métodos não foi descrita e necessita ser investigada.
$\mathrm{O}$ aumento progressivo no estresse mecânico e metabólico tem papel fundamental na otimização dos ganhos de força e hipertrofia muscular proporcionados pelo $\mathrm{TF}^{8}$. Treinos com elevado estresse mecânico (6-12 repetiçôes máximas [RM]) são tradicionalmente recomendados para ganhos ótimos de força e hipertrofia ${ }^{2,3}$. Além disso, recentemente, foi estabelecida uma relação de doseresposta entre o estresse mecânico (quantificado pelo volume de trabalho realizado) e os ganhos hipertróficos(5). O termo estresse metabólico tem sido utilizado para descrever TF que induzam uma maior elevação nos produtos metabólicos ${ }^{8}$. $\mathrm{O}$ estresse metabólico tem sido quantificado por meio da mensuração das respostas do lactato sanguíneo ${ }^{9}$ (LA) e do inchaço muscular ${ }^{10}$, além de também 
ser esperado uma relaçáo proporcional entre o lactato (soluto) e o plasma sanguíneo (solvente) ${ }^{11}$. Evidências têm destacado que o elevado inchaço muscular pode favorecer ganhos hipertróficos ${ }^{12}$. De fato, TF que induzem a um elevado estresse metabólico, porém utilizam baixas cargas (25-35 RM) parecem ser, pelo menos para a hipertrofia, igualmente efetivos quando comparados a treinos com altas cargas (8-12 RM) ${ }^{13}$. Assim, métodos de TF que induzem um maior volume de trabalho, bem como um maior estresse metabólico seriam potencialmente favoráveis para promover ganhos de força e hipertrofia muscular.

Uma estratégia que vem sendo utilizada no TF para aumentar o estresse mecânico é o alongamento estático realizado nos intervalos de recuperação entre as séries. Recentemente, Hany Rambod, ex-fisiculturista, desenvolveu um método de TF conhecido como facial streching training-7 (FST7). Nesse método, o praticante realiza 7 séries de

\section{Método}

\section{Voluntários}

A amostra foi composta por 10 homens com experiência em TF (TABELA 1). Todos os voluntários estavam cientes quanto aos procedimentos do estudo, benefícios e riscos antes da assinatura do termo de consentimento livre e esclarecido (TCLE). O estudo foi aprovado pelo Comitê de Ética em Pesquisa com Seres Humanos Institucional (parecer no 4775/2017).

\section{Delineamento do estudo}

Os voluntários realizaram quatro visitas ao laboratório, sendo uma para avaliação dos critérios de inclusão e familiarização, e as demais para a realização das sessóes experimentais. Todas as visitas foram em um mesmo período do dia. Na primeira visita, foram preenchidos os questionários de anamnese, assinatura do TCLE, e medidas de massa corporal e estatura. A seguir, foi realizada uma familiarização com o dinamômetro isocinético. Os voluntários foram submetidos a três protocolos, sendo: 1) facial streching training-7 (FST-7), 2) Controle (CON), e 3) tradicional (TRAD). A ordem de realizaçáo dos protocolos foi aleatória (www.randomization.com) com intervalo mínimo de $72 \mathrm{~h}$ entre as sessóes ${ }^{19}$.
8-12 repetiçóes com 30 s de intervalo, sendo cerca de $20 \mathrm{~s}$ de alongamento estático da musculatura agonista. Evidências, porém, têm demonstrado que o alongamento pode reduzir o desempenho neuromuscular ${ }^{14} \mathrm{e}$ atenuar os ganhos hipertróficos ${ }^{15}$, 16. De fato, o alongamento entre as séries pode reduzir a velocidade de execução do movimento e o trabalho total ${ }^{17}$, além de aumentar a percepção de esforço da sessão de $\mathrm{TF}^{18}$.

Considerando que o estresse mecânico e metabólico imposto pelo método FST-7 ainda não são conhecidos, o objetivo do presente estudo é investigar os efeitos agudos do FST-7 no trabalho total, índice de fadiga, espessura muscular (EM), e LA de homens experientes no TF. Nós hipotetizamos que o FST-7 causará uma redução mais pronunciada na capacidade do músculo de gerar força, refletida pelo menor trabalho total e maior índice de fadiga, enquanto produzirá menores aumentos na EM e no LA.

\section{Protocolos de exercícios}

Os protocolos foram realizados no dinamômetro isocinético (Biodex 4, Medical, Inc., Shirley, NY). Os voluntários realizaram a extensão concêntrica unilateral do joelho a $60^{\circ} / \mathrm{s} \mathrm{com} 75^{\circ}$ de amplitude do movimento. A posição inicial do movimento foi a $90^{\circ}$ de flexão do joelho e a posição final foi de $15^{\circ}$ de flexão do joelho. Os voluntários foram posicionados na cadeira do dinamômetro com os quadris e tronco imobilizados por meio de cintas, para minimizar movimentos compensatórios. Os voluntários foram incentivados verbalmente, pelo mesmo pesquisador, a realizarem a maior força possível em todas as repetiçóes. Os protocolos de exercícios foram: 1) FST-7 (sete séries de 10 repetiçóes de extensão unilateral de joelho com $30 \mathrm{~s}$ de intervalo entre as séries, sendo $20-25$ s de alongamento do quadríceps; 2) CON (sete séries de 10 repetiçóes de extensão unilateral de joelho com $30 \mathrm{~s}$ de intervalo entre as séries sem alongamento); e 3) TRAD (sete séries de 10 repetições de extensão unilateral de joelho com 120 $s$ de intervalo entre as séries sem alongamento). $\mathrm{O}$ trabalho total consistiu no somatório do que foi realizado nas sete séries de 10 repetiçôes (joules). $\mathrm{O}$ índice de fadiga (IF) foi calculado pela queda 
percentual no pico de torque $(\mathrm{PT})$ entre a primeira e a última série, sendo: $\mathrm{IF}=[(\mathrm{PT} 7 \mathrm{7}$ série $/ \mathrm{PT}$ $1^{\text {a }}$ série $\left.{ }^{*} 100\right]$. O erro típico das medidas do isocinético a $60^{\circ} / \mathrm{s}$ são de $12 \mathrm{~N} \cdot \mathrm{m}$ e o coeficiente de variação de $4,6 \%{ }^{20}$.

\section{Espessura muscular}

Um ultrassom B-Mode (Philips-VMI, Ultra Vision Flip, model BF; Lagoa Santa, MG, Brazil) foi utilizado para mensurar a espessura dos músculos reto femoral e vasto intermédio antes e imediatamente após o exercício. O transdutor (10 $\mathrm{MHz}$ ) foi posicionado transversalmente sobre a pele e as medidas foram realizadas com o voluntário em decúbito dorsal, após $5 \mathrm{~min}$ de repouso. A medida foi obtida a $20 \%$ da distância entre a borda superior da patela e a espinha ilíaca anterossuperior ${ }^{21}$. Uma camada de gel hidrossolúvel foi aplicada na superfície do transdutor para fornecer transmissão acústica apropriada e evitar a compressão dos tecidos subjacentes. A EM foi mensurada considerando a distância entre a borda superior do fêmur e a fáscia mais superficial do reto femoral (FIGURA 1). O erro típico da medida de EM é de $0,96 \mathrm{~mm}$ e o coeficiente de variaçáo de $2,4 \%{ }^{20}$.

\section{Lactato sanguíneo}

As amostras sanguíneas foram coletadas no lóbulo da orelha direita. Para tal, foi realizada a limpeza do local com algodão umedecido em álcool etílico a 70\%, massageando o lóbulo para estimular a perfusão sanguínea. A seguir, utilizando luvas de procedimento e lanceta descartáveis foi realizada uma punçáo para coleta de $25 \mu \mathrm{l}$ de sangue com tubo capilar descartável. As amostras foram depositadas em microtúbulos (Eppendorf ${ }^{\oplus}$ ) contendo $50 \mu \mathrm{de}$ soluçáo de fluoreto de sódio a [1\%]. As amostras foram armazenadas em freezer $\left(-20^{\circ} \mathrm{C}\right)$ até o momento da análise. Foram realizadas coletas antes e após três minutos do término dos três protocolos ${ }^{3}$. O LA foi quantificado pelo lactímetro YSI 1500 (Yellow Springs Instrument, OH, USA). O erro típico de medida do LA de repouso é de $0,1 \mathrm{mMol} / \mathrm{L}$ e o coeficiente de variação é de $15,6 \%{ }^{20}$.

\section{Alongamento do FST-7}

Para o alongamento do quadríceps, os voluntários posicionaram a perna sobre um banco e sentaram sobre o calcanhar. Para aumentar a intensidade do alongamento o voluntário realizava extensão do quadril até a máxima amplitude tolerável. O pé contralateral foi mantido no chão com o joelho estendido (FIGURA 2).

\section{Análise estatística}

Os dados apresentam distribuição normal e estấo reportados segundo a estatística descritiva média e desvio padrão. A comparação entre as modificaçóes no trabalho total, índice de fadiga e espessura muscular induzida pelo exercício foram realizadas por meio da ANOVA one-way para medidas repetidas, enquanto que a ANOVA de modelos mistos foi utilizada nas comparaçóes pré e pós exercício e entre os três protocolos. O teste post hoc adotado foi o de Bonferroni, quando observado diferença significativa. O nível de confiança considerado foi de 0,05 e o software utilizado foi o Statistical Package Social Science (IBM SPSS, versão $20 \mathrm{NY}$, USA).

TABELA 1 - Característica dos voluntários do estudo $(\mathrm{n}=10)$.

\begin{tabular}{lc}
\hline Características & Média \pm Desvio-padrão \\
\hline Idade (anos) & $27,0 \pm 6,2$ \\
Massa corporal $(\mathrm{kg})$ & $81,8 \pm 23,4$ \\
Estatura $(\mathrm{cm})$ & $178,1 \pm 8,6$ \\
Experiência no treinamento de força (anos) & $5,6 \pm 3,7$ \\
Torque dos extensores do joelho a 60\% $(\mathrm{N} \cdot \mathrm{m})$ & $263,5 \pm 67,7$ \\
\hline
\end{tabular}

Rev Bras Educ Fís Esporte, (São Paulo) 2020 J ul-Set;34(3):437-45 • 439 


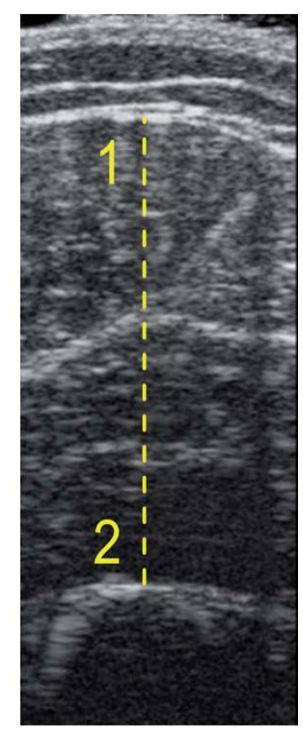

FIGURA 1 - Imagem representativa de um participante. A linha pontilhada representa a espessura muscular do extensores do joelho, definido como a distância entre a fáscia superficial do reto femoral (1) e a borda superior do fêmur (2).
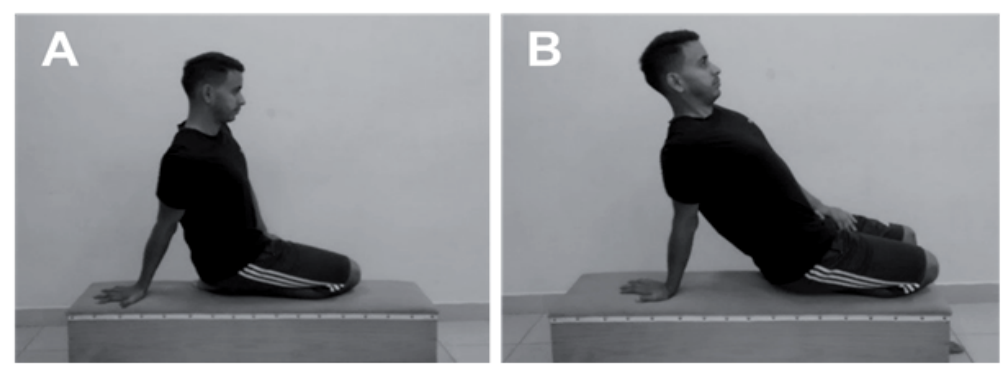

FIGURA 2 - Alongamento estático do quadríceps realizado durante o protocolo FST-7. A: posição inicial, B: posição do alongamento mantida por 20-25 s.

\section{Resultados}

O trabalho total realizado no protocolo FST-7 $(9.361 \pm 1.714 \mathrm{~J})$ foi menor $(\mathrm{p}<0,001)$ do que no CON $(10.730 \pm 2.360 \mathrm{~J})$ e no TRAD $(12.160 \pm 2.200 \mathrm{~J})$, bem como o trabalho total no CON foi menor $(\mathrm{p}<0,001)$ do que no TRAD (FIGURA 3).

O índice de fadiga no FST-7 $(38,5 \pm 13,4 \%)$ foi maior do que no CON $(29,0 \pm 10,9 \%)$ e no TRAD $(12,0 \pm 10,4 \%)(p<0,001)$. Ainda, no CON $(29,0 \pm$ $10,9 \%)$ o índice de fadiga foi maior do que no TRAD $(12,0 \pm 10,4 \%)(\mathrm{p}<0,001)($ FIGURA 4).
$\mathrm{O}$ aumento na EM foi similar entre os protocolos FST-7 (27,6 $\pm 14,3 \%)$, CON $(27,2 \pm 11,6 \%)$ e TRAD $(33,2 \pm 9,5 \%)(p>0,05)($ FIGURA 5$)$.

O LA no repouso foi similar $(p>0,05)$ entre os protocolos FST-7 $(1,1 \pm 0,7 \mathrm{mMol} / \mathrm{L}), \mathrm{CON}(0,8 \pm$ $0,3 \mathrm{mMol} / \mathrm{L})$ e TRAD $(1,0 \pm 0,4 \mathrm{mMol} / \mathrm{L})$. Houve um aumento $(\mathrm{p}<0,05)$ no LA 3 min após o exercício, porém sem diferença $(\mathrm{p}>0,05)$ entre os protocolos FST-7 $(4,5 \pm 1,5 \mathrm{mMol} / \mathrm{L}), \mathrm{CON}(4,4 \pm 0,8 \mathrm{mMol} / \mathrm{L})$ e TRAD (4,6 $\pm 1,3 \mathrm{mMol} / \mathrm{L})$ (FIGURA 6). 


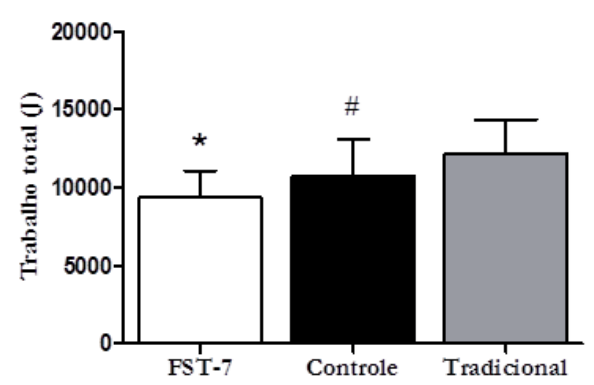

* $p<0,05$ menor que Controle e Tradicional. $\# p<0,05$ menor que Tradicional.

FIGURA 3 - Comparação do trabalho total (J oule - J) dos extensores do joelho nos protocolos FST-7, Controle e Tradicional.

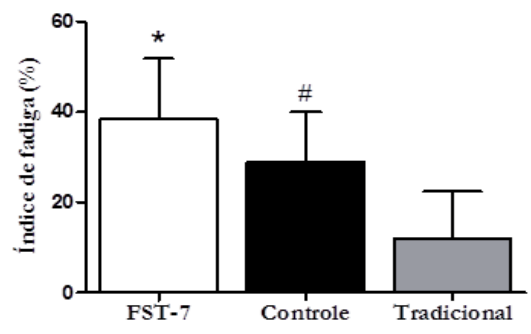

${ }^{*} p<0,05$ maior que Controle e Tradicional; $\# p<0,05$ maior que Tradicional.

FIGURA 4 - Índice de fadiga nos protocolos FST-7, Controle e Tradicional.

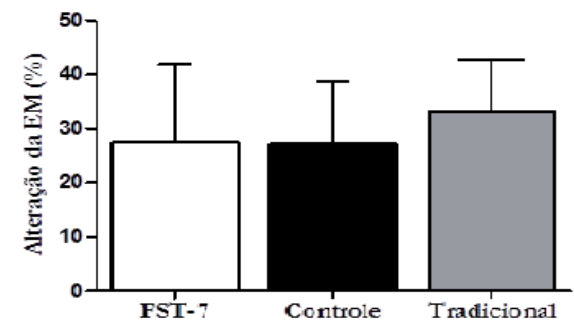

FIGURA 5 - Aumento percentual da espessura muscular (EM) nos protocolos FST-7, Controle e Tradicional em resposta ao exercício de extensão de joelhos.

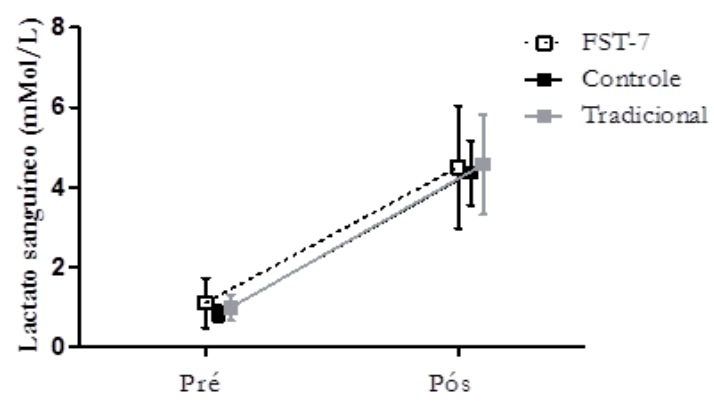

FIGURA 6 - Lactato sanguíneo no repouso e três minutos após a sessão de exercício de extensão de joelhos nos protocolos FST-7, Controle e Tradicional. 


\section{Discussão}

O objetivo do presente estudo foi avaliar os efeitos agudos do FST-7 nas respostas neuromusculares (trabalho total e índice de fadiga) e metabólicas (inchaço por meio da EM e LA) de homens experientes no TF. Os resultados mostram que o FST-7 apresentou um maior índice de fadiga, além de uma menor capacidade de produçáo de trabalho total comparado com CON e TRAD. Em relação a EM e o LA, não foi observada diferença significativa entre os protocolos. Dessa forma, parece que o FST-7 diminui a capacidade de produzir força muscular agudamente com aumentos na EM e LA semelhantes a métodos de TF tradicionais.

Estudos prévios demonstraram que o alongamento estático antes ou entre as séries do TF diminui a capacidade de produção de força ${ }^{14-16,18,22}$. A diminuição aguda da força pode ser atribuída a fatores centrais (reduzido drive central) e periféricos (reduzido processo de excitação-contração) $)^{14,16,23-25}$.

Neste estudo observamos que o FST-7 induziu a um maior índice de fadiga, bem como um menor trabalho total quando comparado ao CON e TRAD. Portanto, a redução no desempenho pode ser atribuída ao alongamento entre as séries. As diferenças entre FST-7 e TRAD também podem ser atribuídas ao menor intervalo de recuperação que é utilizado no FST-7. É bem estabelecido que o menor intervalo de recuperação entre as séries diminui a restauração do sistema fosfagênico ${ }^{26} \mathrm{e}$, portanto justificaria as diferenças entre os protocolos com menor e maior intervalo de recuperação entre as séries.

Apesar de alguns estudos mostrarem que alongamento inferior a $45 \mathrm{~s}$ não afetaria a capacidade do músculo de produzir força ${ }^{27-29}$, no presente estudo, o FST-7 apresentou maior índice de fadiga do que o CON, evidenciando que o alongamento estático de 20 - 25 s entre séries diminui a capacidade do músculo em produzir força nas séries subsequentes. O maior índice de fadiga no FST-7 pode ser explicado por fatores centrais periféricos e periféricos ${ }^{14}$, ambos responsáveis por diminuírem agudamente a produção de força muscular ${ }^{14,15}$. O alongamento estático diminui a sensitividade dos fusos musculares, levando a diminuiçáo da atividade das fibras aferentes de grande calibre ${ }^{30}$. O alongamento pode também reduzir a excitabilidade dos motoneurônios alfa por estimular os receptores articulares tipos III e $\mathrm{IV}^{30}$. Além disso, o alongamento pode modificar propriedades viscoelásticas da unidade miotendinosa ${ }^{30}$. Nesse caso, tanto a tensão passiva quanto a rigidez miotendinosa podem ter sido reduzidas, assim, diminuindo a transferência de tensão para o osso, resultando em menor produção de torque ${ }^{31}$. O menor índice de fadiga no TRAD em relação ao CON e FST-7 pode ser atribuído ao maior intervalo de recuperação entre as séries. Maior intervalo promoveria maior fluxo sanguíneo local e maior restauração do sistema fosfagênico, favorecendo a capacidade do músculo em produzir força nas séries subsequentes ${ }^{32,33}$.

Foi hipotetizado que o FST-7 induziria um maior aumento da EM. Também poderia ser esperado um maior aumento no LA no FST-7 comparado a outros métodos de TF. Essa maior concentração de LA teria um efeito direto sobre a EM, uma vez que a maior concentraçáo de solutos induziria uma maior $\mathrm{EM}^{11}$. Porém, neste trabalho o LA e a EM aumentaram similarmente entre os três protocolos, isso sugere que o aumento da tensão mecânica por meio do alongamento estático entre as séries não foi suficiente para elevar o estresse metabólico no FST-7 em comparação aos demais protocolos utilizados. Interessantemente, o menor intervalo de recuperação entre as séries utilizados no FST-7 e no $\mathrm{CON}$ também não foram suficientes para induzir uma maior concentração de LA. Estes resultados, talvez, sejam explicados pelo menor trabalho total realizado nos protocolos com menor intervalo de recuperaçáo entre as séries quando comparado ao TRAD com $120 \mathrm{~s}$ de intervalo de recuperação.

Esse é o primeiro estudo sobre o FST-7, portanto, comparações específicas ainda não são possíveis. Contudo, os resultados deste estudo sugerem que as adaptaçóes metabólicas do FST-7 não são diferentes das ocorridas em outros métodos de TF tradicionais (p. ex., CON e TRAD). É importante destacar que este estudo investigou apenas os efeitos agudos do FST-7 e estudos crônicos são necessários. Porém, uma evidência recente sugere que o alongamento atenue o efeito hipertrófico do TF. Junior et al. ${ }^{16}$ investigaram os efeitos de duas séries de alongamento estático, sendo cada série de $25 \mathrm{~s}$ de duração, as quais foram realizadas imediatamente antes da sessão de TF. Embora o alongamento tenha promovido melhoria na flexibilidade e não tenha afetado os ganhos de força, os ganhos hipertróficos foram atenuados pelo alongamento. Logo, poderia ser hipotetizado que cronicamente o FST-7 também atenuaria os ganhos hipertrófico, similar ao que foi observado por JUnior et al. ${ }^{16}$. 
O presente estudo apresenta a limitação de ter realizado apenas uma coleta de sangue para quantificar o LA 3-min após os três protocolos de exercícios resistidos. Todavia, acreditamos que medidas adicionais ou em diferente momento, provavelmente, produziria resultados similares ${ }^{34}$.
Portanto, conclui-se que embora o FST-7 induza aumentos similares na EM e LA, este método reduziu o trabalho total e ocasionou maior índice de fadiga. Este estudo fornece evidências que o FST-7, talvez, não seja um método de TF adequado para induzir ganhos ótimos de força e hipertrofia muscular.

\begin{abstract}
Neuromuscular and metabolic responses from fst-7 strength training method in trained men

The aim of the study was to assess neuromuscular and metabolic acute responses of FST-7 resistance training protocol. Ten trained men were submitted to three resistance exercise (RE) protocols. 1) FST-7 (seven sets of 10 repetitions of isokinetic knee extensions with $30 \mathrm{sec}$ of rest interval between sets, including 20 - $25 \mathrm{sec}$ of quadriceps stretching; 2) Control (CON, seven sets of 10 repetitions of isokinetic knee extensions with 30 sec of rest interval between sets, without stretching); and 3) Traditional (TRAD seven sets of 10 repetitions of isokinetic knee extensions with $120 \mathrm{sec}$ of rest interval between sets, without stretching). Total work performed during TRAD $(12.160 \pm 2.200 \mathrm{~J})$ was greater than FST-7 $(9.361 \pm 1.714 \mathrm{~J})$ and CON $(10.730 \pm 2.360 \mathrm{~J})$, CON also produced greater total work than FST-7 ( $p<$ $0.05)$. The decrement in work along sets (fatigue index) was greater in FST-7 (38.5 $\pm 13.4 \%)$ compared with CON $(29.0 \pm 10.9 \%)$ and TRAD $(12.0 \pm 10.4 \%)$. Muscle swelling and blood lactate significantly increased ( $p>0.05$ ), however, no difference between protocols were found. These results suggest that FST-7 presents similar metabolic responses to CON and TRAD, but TRAD and CON induce greater mechanical overload than FST-7.
\end{abstract}

KEYwoRDs: Resistance exercise; Stretching; Muscle hyperemia; Fatigue index; Blood lactate.

\title{
Referências
}

1. Suchomel TJ, Nimphius S, Stone MH. The importance of muscular strength in athletic performance. Sports Med. 2016;46(10):1419-49.

2. Kraemer WJ, Adams K, Cafarelli E, Dudley GA, Dooly C, Feigenbaum MS, et al. American College of Sports Medicine position stand. Progression models in resistance training for healthy adults. Med Sci Sports Exerc. 2002;34(2):364-80.

3. Medicine ACS. American College of Sports Medicine position stand. Progression models in resistance training for healthy adults. Med Sci Sports Exerc. 2009;41(3):687-708.

4. Gentil P, Oliveira E, Fontana K, Molina G, Oliveira RJ, Bottaro M. Efeitos agudos de vários métodos de treinamento de força no lactato sanguíneo e características de cargas em homens treinados recreacionalmente. Rev Bras Med Esporte. 2006;12(6):303-7.

5. Schoenfeld BJ, Ogborn D, Krieger JW. Dose-response relationship between weekly resistance training volume and increases in muscle mass: A systematic review and meta-analysis. J Sports Sci. 2016:1-10.

6. Bottaro M, Veloso J, Wagner D, Gentil P. Resistance training for strength and muscle thickness: Effects of number of sets and muscle group trained. Sci Sports. 2011;26:259-64.

7. Krieger JW. Single vs. multiple sets of resistance exercise for muscle hypertrophy: a meta-analysis. J Strength Cond Res. 2010;24(4):1150-9.

8. Schoenfeld BJ. The mechanisms of muscle hypertrophy and their application to resistance training. J Strength Cond Res. 2010;24(10):2857-72.

9. Goto K, Nagasawa M, Yanagisawa O, Kizuka T, Ishii N, Takamatsu K. Muscular adaptations to combinations of high- and low-intensity resistance exercises. J Strength Cond Res. 2004;18(4):730-7. 
10. Jenkins ND, Housh TJ, Bergstrom HC, Cochrane KC, Hill EC, Smith CM, et al. Muscle activation during three sets to failure at 80 vs. 30\% 1RM resistance exercise. Eur J Appl Physiol. 2015;115(11):2335-47.

11. Ploutz-Snyder LL, Convertino VA, Dudley GA. Resistance exercise-induced fluid shifts: change in active muscle size and plasma volume. Am J Physiol. 1995;269(3 Pt 2):R536-43.

12. Loenneke JP, Fahs CA, Wilson JM, Bemben MG. Blood flow restriction: the metabolite/volume threshold theory. Med Hypotheses. 2011;77(5):748-52.

13. Schoenfeld BJ, Peterson MD, Ogborn D, Contreras B, Sonmez GT. Effects of low- vs. high-load resistance training on muscle strength and hypertrophy in well-trained men. J Strength Cond Res. 2015;29(10):2954-63.

14. Trajano GS, Seitz L, Nosaka K, Blazevich AJ. Contribution of central vs. peripheral factors to the force loss induced by passive stretch of the human plantar flexors. J Appl Physiol (1985). 2013;115(2):212-8.

15. Trajano GS, Nosaka K, B Seitz L, Blazevich AJ. Intermittent stretch reduces force and central drive more than continuous stretch. Med Sci Sports Exerc. 2014;46(5):902-10.

16. Junior RM, Berton R, de Souza TM, Chacon-Mikahil MP, Cavaglieri CR. Effect of the flexibility training performed immediately before resistance training on muscle hypertrophy, maximum strength and flexibility. Eur J Appl Physiol. 2017;117(4):767-74.

17. García-López D, Izquierdo M, Rodríguez S, González-Calvo G, Sainz N, Abadía O, et al. Interset stretching does not influence the kinematic profile of consecutive bench-press sets. J Strength Cond Res. 2010;24(5):1361-8.

18. Souza ACR, Bastos CLB, Portal MND, Salles BF, Gomes TM, Novaes JF. Efeito agudo do intervalo passivo e do alongamento no desempenho de séries múltiplas. Rev Bras Cineantrop Desempenho Hum. 2009;11(4):435-43.

19. Soares S, Ferreira-Junior JB, Pereira MC, Cleto VA, Castanheira RP, Cadore EL, et al. Dissociated Time Course of Muscle Damage Recovery Between Single- and Multi-Joint Exercises in Highly Resistance-Trained Men. J Strength Cond Res. 2015;29(9):2594-9.

20. Hopkins WG. Measures of reliability in sports medicine and science. Sports Med. 2000;30(1):1-15.

21. Coutinho EL, Gomes AR, França CN, Oishi J, Salvini TF. Effect of passive stretching on the immobilized soleus muscle fiber morphology. Braz J Med Biol Res. 2004;37(12):1853-61.

22. Souza AC, Bentes CM, de Salles BF, Reis VM, Alves JV, Miranda H, et al. Influence of inter-set stretching on strength, flexibility and hormonal adaptations. J Hum Kinet. 2013;36:127-35.

23. Opplert J, Genty JB, Babault N. Do stretch durations affect muscle mechanical and neurophysiological properties? Int J Sports Med. 2016;37(9):673-9.

24. Marek SM, Cramer JT, Fincher AL, Massey LL, Dangelmaier SM, Purkayastha S, et al. Acute effects of static and proprioceptive neuromuscular facilitation stretching on muscle strength and power output. J Athl Train. 2005;40(2):94103.

25. Cramer JT, Housh TJ, Johnson GO, Miller JM, Coburn JW, Beck TW. Acute effects of static stretching on peak torque in women. J Strength Cond Res. 2004;18(2):236-41.

26. Weir J, Wagner L, Housh T. The effects of rest interval length on repeated maximal bench presses. J Strength Cond Res. 1994;8(1):58-60.

27. Kay AD, Blazecich AJ. Reductions in active plantarflexor moment are significantly correlated with static stretch duration. European Journal of Sport Science. 2008;8(1):41-6.

28. Siatras TA, Mittas VP, Mameletzi DN, Vamvakoudis EA. The duration of the inhibitory effects with static stretching on quadriceps peak torque production. J Strength Cond Res. 2008;22(1):40-6.

29. Knudson D, Noffal G. Time course of stretch-induced isometric strength deficits. Eur J Appl Physiol. 2005;94(3):34851.

30. Avela J, Kyröläinen H, Komi PV. Altered reflex sensitivity after repeated and prolonged passive muscle stretching. J Appl Physiol (1985). 1999;86(4):1283-91.

31. Kubo K, Kanehisa H, Kawakami Y, Fukunaga T. Influence of static stretching on viscoelastic properties of human tendon structures in vivo. J Appl Physiol (1985). 2001;90(2):520-7.

32. Enoka RM, Duchateau J. Muscle fatigue: what, why and how it influences muscle function. J Physiol. 2008;586(1):1123.

33. McCartney N. Acute responses to resistance training and safety. Med Sci Sports Exerc. 1999;31(1):31-7.

34. Wirtz N, Wahl P, Kleinöder H, Mester J. Lactate kinetics during multiple set resistance exercise. J Sports Sci Med. 2014;13(1):73-7. 
ENDEREÇO

Martim Bottaro

Faculdade de Educação Física

Universidade de Brasília Asa Norte, s/n

70919-970 - Brasília - DF - Brasil

E-mail: martim@unb.br, martim.bottaro@gmail.com
Submetido: 28/06/2017

1a revisão: 09/09/2018

$2^{\text {a }}$ revisão: $05 / 12 / 2017$

Aceito: 01/ 02/2018 